University of Nebraska - Lincoln

DigitalCommons@University of Nebraska - Lincoln

2005

\title{
Chemical mapping of polymer photoresists by scanning transmission $\mathrm{x}$-ray microscopy
}

\author{
Ligia Muntean \\ University of California - Berkeley \\ Romain Planques \\ University of California - Berkeley
}

A. L. D. Kilcoyne

Lawrence Berkeley National Laboratory, ALKilcoyne@lbl.gov

Stephen R. Leone

University of California - Berkeley, srl@berkeley.edu

Mary Gilles

Lawrence Berkeley National Laboratory, mkgilles@lbl.gov

See next page for additional authors

Follow this and additional works at: https://digitalcommons.unl.edu/usdoepub

Part of the Bioresource and Agricultural Engineering Commons

Muntean, Ligia; Planques, Romain; Kilcoyne, A. L. D.; Leone, Stephen R.; Gilles, Mary; and Hinsberg, William D., "Chemical mapping of polymer photoresists by scanning transmission $x$-ray microscopy" (2005). US Department of Energy Publications. 340.

https://digitalcommons.unl.edu/usdoepub/340

This Article is brought to you for free and open access by the U.S. Department of Energy at DigitalCommons@University of Nebraska - Lincoln. It has been accepted for inclusion in US Department of Energy Publications by an authorized administrator of DigitalCommons@University of Nebraska - Lincoln. 


\section{Authors}

Ligia Muntean, Romain Planques, A. L. D. Kilcoyne, Stephen R. Leone, Mary Gilles, and William D. Hinsberg 


\title{
Chemical mapping of polymer photoresists by scanning transmission $\mathrm{x}$-ray microscopy
}

\author{
Ligia Muntean and Romain Planques ${ }^{\mathrm{a})}$ \\ Department of Chemistry and Physics, University of California, Berkeley, California 94720
}

A. L. D. Kilcoyne

Advanced Light Source, Lawrence Berkeley National Laboratory, Berkeley, California 94720

Stephen R. Leone

Department of Chemistry and Physics, University of California, Berkeley, California 94720

and Chemical Sciences Division, Lawrence Berkeley National Laboratory, Berkeley, California 94720

Mary K. Gilles ${ }^{\text {b) }}$

Chemical Sciences Division, Lawrence Berkeley National Laboratory, Berkeley, California 94720

William D. Hinsberg

IBM Almaden Research Center, 650 Harry Road, San Jose, California 95120

(Received 14 April 2005; accepted 23 May 2005; published 22 July 2005)

\begin{abstract}
Scanning transmission x-ray microscopy (STXM) is shown to be a powerful imaging technique that provides chemical selectivity and high spatial resolution $(\sim 35 \mathrm{~nm})$ for studying chemically amplified photoresists. Samples of poly(4-t-butoxycarbonyloxystyrene) PTBOCST resist, imprinted by deep ultraviolet lithography with a line/space pattern of $1.10 \mu \mathrm{m} / 0.87 \mu \mathrm{m}$ followed by a post-exposure bake, are used to demonstrate STXM imaging capabilities to extract photoresist latent images. Chemical contrast is obtained by measuring the x-ray absorption at an energy of $290.5 \mathrm{eV}$, corresponding to a carbon $K$ shell electronic transition to the unoccupied $\pi^{*}$ molecular orbital of the PTBOCST carbonyl group. A quantitative analysis provides the spatial distribution of the fraction of the unexposed and deprotected polymers remaining after the post-exposure bake stage as well as the thickness of both regions. Both chemical and topographical contributions to the total contrast are estimated. Advantages and limitations of STXM in comparison with other imaging techniques with chemical specificity are discussed. (0) 2005 American Vacuum Society. [DOI: 10.1116/1.1978899]
\end{abstract}

\section{INTRODUCTION}

The continuing increase of integrated circuit performance is largely based on the capability of advanced lithographic techniques to pattern resist materials with sub-100 $\mathrm{nm}$ line dimensions. ${ }^{1-5}$ With decreasing feature sizes, new demands are placed on measurement characterization techniques at different stages of pattern formation.

The process of transferring a pattern to semiconductor surfaces by chemically amplified resist lithography consists of several steps. A latent image is first created by exposing the photoresist to short-wavelength (typically UV) radiation through a patterned mask. For test patterns, interferometric lithography can also be used to create a sinusoidal standing wave pattern produced by interference at the region of intersection of two coherent light beams. ${ }^{6}$ An acid species is produced in the photoresist (an acid-labile polymer blended with a photo acid generator) only in the regions exposed to radiation, creating a first latent image. The exposure stage is followed by a complex reaction-diffusion process that takes place during a post-exposure bake at elevated temperatures. During the bake the acid diffuses and interacts with multiple reaction sites to catalyze deprotection, which alters the

\footnotetext{
${ }^{\text {a) }}$ Present address: Ecole Normale Supérieure, 75005 Paris, France.

${ }^{\text {b) }}$ Author to whom all correspondence should be addressed; electronic mail: MKGilles@lbl.gov
}

chemical and physical properties of the polymer. These chemical changes provide routes to investigate polymer photochemistry and measure line dimensions of latent images at this important intermediate stage in the image formation. The final step is the development of the latent image by dissolution of either the deprotected (positive tone resist) or unmodified polymer (negative tone resist) areas followed by selective etching of the underlying semiconductor wafer in the regions where the polymer was removed.

Of primary importance for controlling line dimensions in the sub-100 $\mathrm{nm}$ range is the measurement of the spatial extent of deprotection, which is influenced by the spatial evolution of the reaction-diffusion front. To achieve this goal, chemically sensitive techniques with the potential for high spatial resolution are needed. Fluorescence microscopy is used to characterize polymer photoresists through specific labeling with $\mathrm{pH}$ sensitive dyes. ${ }^{7,8}$ Chemical contrast images (due to selective absorption) have been obtained with a spatial resolution of $300 \mathrm{~nm}$ at $3 \mu \mathrm{m}$ (the $\mathrm{OH}$ stretching vibration) along with a simultaneous topographical image by using infrared near field scanning optical microscopy (IR NSOM). ${ }^{9-12}$ Considerably higher spatial resolution is expected to be achieved by apertureless near field scanning optical microscopy when used with infrared absorption contrast. ${ }^{13,14}$ However, this method also faces significant challenges in detecting the small signal (light scattered from 
the tip) in the presence of a large background. Coherent antiStokes Raman scattering (CARS) microscopy was used for imaging polymer photoresists with a spatial resolution of 270 $\mathrm{nm}$ by probing the carbonyl stretching vibration in samples of poly(tert-butoxycarbonyloxystyrene). ${ }^{15}$ Using complementary x-ray and neutron reflectivity measurements, nonimaging techniques can detect the spatial evolution of the reaction-diffusion process in chemically amplified photoresists with extremely high resolution (in the range of a few nanometers). These complex measurements yield compositional and density profiles through isotopic labeling. ${ }^{16}$

In this article, we explore the potential to use scanning transmission x-ray microscopy (STXM) for the rapid chemical imaging of polymer photoresists. STXM, ${ }^{17-20}$ currently a synchrotron radiation soft $\mathrm{x}$-ray-based technique, has advanced rapidly in the last decade. In this study, we demonstrate the chemical sensitivity of STXM by examining line patterns in poly(tert-butoxycarbonyloxystyrene) (PTBOCST) polymer photoresists at the carbon $K$ edge. We provide a quantitative chemical mapping of the relevant chemical species, which yields important information about the spatial extent of the deprotection reaction. In addition, this quantitative analysis provides independent values of the thickness of the two polymer regions. The application of STXM to polymer photoresists is of primary importance due to its high spatial resolution $(35 \mathrm{~nm})$. Furthermore, the technique has a great potential and opens unique modalities for future studies of the reaction-diffusion process due to the short acquisition times, the excellent spatial resolution, and the development of experimental setups which enable measurements of samples placed in heating cells and under chemical exposure.

\section{STXM TECHNIQUE}

\section{A. Achieving high spectral resolution}

Since STXM has not been applied to analyze patterned polymer photoresists, a brief description of the basic method of scanning transmission $\mathrm{x}$-ray microscopy is provided here.

The absorption of $\mathrm{x}$ rays passing through the sample depends on the nature of the substance, the thickness of the sample and the density of absorbing centers. Photon absorption can cause excitations of inner shell electrons to unoccupied energy levels or can remove the electron completely to generate an ionized atom or molecule. Traditionally, x-ray absorption was described in terms of absorption edges, i.e., the onset of inner shell ionization at specific energies for each atom. With the advent of tunable x-ray sources from synchrotron radiation facilities, $\mathrm{x}$-ray absorption spectroscopy became a powerful technique in chemical analysis extending its capabilities beyond providing elemental composition to also providing chemical bonding information. This is due to the increased spectral resolution of soft $\mathrm{x}$ rays produced at synchrotron facilities $(\sim 0.1 \mathrm{eV}$ or better $)$ in comparison to the spectral resolution obtained with hard $\mathrm{x}$ rays $(\sim 1 \mathrm{eV})$. The sharp spectral features observed close to the absorption edge are referred to as "near edge x-ray absorption fine structure" (NEXAFS). ${ }^{21,22}$ These features, which can be much stronger than the absorption edge change itself, correspond to transitions of the inner-shell electron to unfilled molecular orbitals or conduction bands. As the x-ray energy is increased throughout an absorption edge, the first observed structure is often associated with the lowest unoccupied molecular orbital, a $\pi^{*}$ orbital for unsaturated molecules (double or triple bonds), followed by transitions to higher energy unoccupied molecular orbitals, typically of $\sigma^{*}$ character associated with saturated (single) chemical bonds, and finally direct inner-shell ionization. ${ }^{17,18,21}$ This fine structure is determined by the geometric and electronic bonding structure of the sample. NEXAFS spectra can be significantly different even for similar chemical compounds. Hence, NEXAFS spectra can often be used as a fingerprint for distinguishing between very similar compounds. We use this capability in the following sections to distinguish between the initial and chemically transformed polymer photoresist materials.

\section{B. Concept of STXM}

The incident synchrotron soft $\mathrm{x}$ rays are monochromated, then focused to a diffraction-limited spot (typically $35 \mathrm{~nm}$ ) by a Fresnel zone plate. Images are obtained by measuring the intensity of the transmitted $\mathrm{x}$ rays as the sample is raster scanned through the focused beam. Spectra are recorded in point, line, or image mode by acquiring signals at multiple photon energies. Spectra at each pixel are measured by recording images as a function of x-ray energy. Images at different energies are acquired individually, or in a stack mode, where the software automatically changes the energy in specified steps and records an image. In the stack mode there are more options available for processing the data (such as the alignment of images at different energies). The signal, converted to optical density (OD), is sensitive to sample thickness, density, and composition, according to the following equation:

$$
\mathrm{OD}=-\ln \left(\frac{I}{I_{0}}\right)=\sigma t=\mu \rho t,
$$

where $I_{0}$ is the incident photon flux intensity, $\sigma$ is the linear absorption coefficient, $t$ is the thickness, $\mu$ is the mass absorption coefficient, and $\rho$ is the density. With too little absorption (sample too thin), the signal is lost in the noise. If the absorption is too high, then various artifacts dominate the observed signal, and the signal loses quantitative accuracy. Normally, the range of OD is from 0.1 to 3 . All samples in this study had optical densities $<1.5$. For carbon studies in organic materials, samples need to be between 50 and 300 $\mathrm{nm}$ thick, a range readily achieved with polymer photoresists.

\section{EXPERIMENTAL METHODS}

The STXM's at beamlines 5.3.2 and 11.0.2 of the Advanced Light Source (Berkeley, CA) were used for these experiments. Beamline 5.3.2 is a horizontal dispersive bending magnet beamline, optimized for an energy range from 


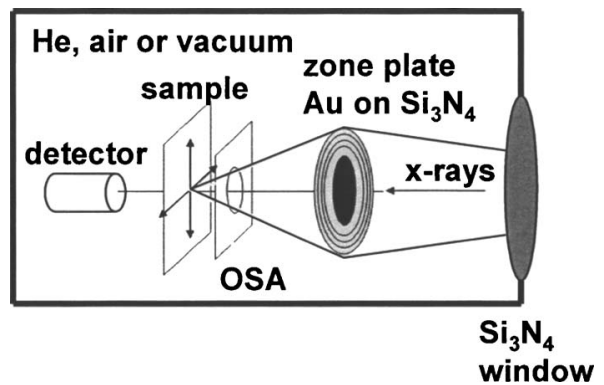

FIG. 1. Schematic of the scanning transmission x-ray microscope (STXM).

250 to $600 \mathrm{eV}$, which covers $\mathrm{C} 1 s, \mathrm{~N} 1 s$, and $\mathrm{O} 1 s$ absorption edges (particularly important for polymer chemistry). However the usable photon range is considerably higher, at least from 200 to $1200 \mathrm{eV}$. The beam is monochromatized by a spherical grating optimized at $\mathrm{C} 1 s$ to give an energy resolution of $100 \mathrm{meV}$. A detailed description of the STXM at 5.3.2 has been published. ${ }^{23}$ Beamline 11.0.2 is an undulator beamline optimized for an energy range from 200 to $1900 \mathrm{eV}$ and operates with a plane grating monochromator consisting of a double ruled grating, with 150 and 1200 lines $/ \mathrm{mm}$. Beamline 11.0.2 has a much higher flux due to the insertion device (elliptically polarizing undulator), hence more care is necessary to prevent radiation damage. This beamline has the potential of providing higher spectral resolution and the possibility of controlling the polarization of the x-ray beam.

The schematic layout of the STXM is shown in Fig. 1. The x-ray beam exits the beamline through a $50 \mathrm{~nm}$ thick $\mathrm{Si}_{3} \mathrm{~N}_{4}$ window, which isolates the STXM chamber from the ultrahigh vacuum of the beamline. The beam is focused by a Fresnel zone plate (provided by the Center for X Ray Optics, Lawrence Berkeley National Laboratory, CA) to a diffraction-limited spot that gives the spatial resolution of the microscope, which for these experiments was typically 35 $\mathrm{nm} .{ }^{24}$ To eliminate undesired diffraction orders at the sample, the zone plate is fabricated with a beam stop at its center. The filtering of the first-order diffracted light is achieved by an order selective aperture, optimally positioned, in combination with the central stop of the zone plate. The diffractionlimited spatial resolution for a fully coherent beam is determined directly by the width of the most outer zone of the zone plate. Zone plates are achromatic lenses with a focal length proportional to the photon energy. Thus, during the acquisition of NEXAFS spectra or an image sequence at many photon energies (referred to as "stacks"), the STXM is refocused synchronously as the photon energy changes. Depending on the focal properties of the zone plate used, this requires motions over $150-200 \mu \mathrm{m}$ for a $30 \mathrm{eV}$ wide $\mathrm{C} 1 s$ NEXAFS scan, or even greater $(>1 \mathrm{~mm})$ for changes between different absorption edges. The STXM's used in this work use interferometer control that allows low distortion imaging and the precise measurement of the transverse position of the zone plate relative to the sample with a precision of $2.5 \mathrm{~nm} .{ }^{24}$ Due to the challenges in scanning x-ray optics, samples are typically scanned relative to the stationary $\mathrm{x}$-ray beam. The sample is scanned using a two-axis $x, y$ piezoelec-

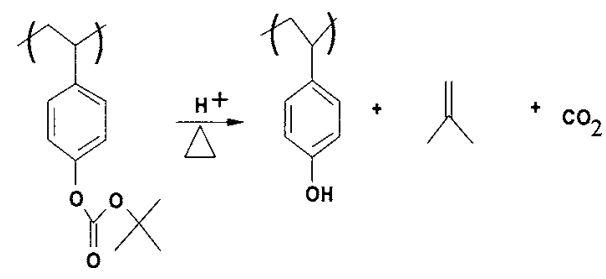

FIG. 2. Schematic representation of the deprotection reaction occurring during the post-exposure bake.

tric stage that provides fine motion over a limited field of view. To provide motion in the range of millimeters, this $x, y$ piezo stage is mounted on top of an $x, y, z$ stepper motor stage. The transmitted $\mathrm{x}$-ray intensity is measured by using a phosphor (fine grained powder on fiber optic) detector that converts $\mathrm{x}$ rays to visible light. The visible light is detected by a high performance photomultiplier with count rate capabilities in the tens of $\mathrm{MHz}$. The software used to process the data is AXIS 2000.

Photoresist samples consisted of thin films of poly(4-tbutoxycarbonyloxystyrene) (PTBOCST) patterned with an unequal line/space pattern having a total period of $\sim 1.97 \mu \mathrm{m}$ (see below). The concentration of the photoacid generator in the resist, bis(4-t-butylphenyl)iodonium perfluorobutanesulfonate, was $0.045 \mathrm{~mol} / \mathrm{kg}$ solids. The pattern was written using UV light through a $100 \mathrm{~nm}$ thick chromium on quartz contact mask with measured opaque chromium regions of $\sim 1.10 \mu \mathrm{m}$ at the top and $1.26 \mu \mathrm{m}$ at the base and open spaces with corresponding dimensions of $0.87 \mu \mathrm{m}$ (top) and $0.71 \mu \mathrm{m}$ (base), respectively. For the first studies using STXM on polymer photoresists, samples with large feature sizes were chosen to demonstrate the chemical contrast in a sample where the spatial resolution would not be a factor. The samples were prepared by coating the resist on 1 in. diameter polished sodium chloride plates followed by a post-apply bake at $130{ }^{\circ} \mathrm{C}$ for $90 \mathrm{~s}$. Following exposure to UV light $\left(10 \mathrm{~mJ} / \mathrm{cm}^{2}\right.$ at $254 \mathrm{~nm}$ for patterned samples, $100 \mathrm{~mJ} / \mathrm{cm}^{2}$ at $254 \mathrm{~nm}$ for blanket exposed control samples), films were post-exposure baked at $100{ }^{\circ} \mathrm{C}$ for $90 \mathrm{~s}$. Under acidic conditions, the tert-butoxylcarbonyl group is converted to a hydroxy group with release of carbon dioxide and iso-butylene as well as other volatile byproducts, as indicated in the chemical formula presented in Fig. 2. Release of reaction byproducts in the exposed regions causes the polymer to shrink, generating a topographical pattern. To lift the polymer off the substrate and mount it for STXM studies, the substrate was then placed in a shallow dish containing deionized water. After approximately 15 min the sodium chloride substrate dissolves and the films float to the surface. These films are placed onto copper TEM grids for the STXM measurements. Small holes in the polymer film, usually present due to preparation procedures, are used to measure the incident photon flux intensity for normalization.

\section{RESULTS AND DISCUSSION}

Figure 3 shows carbon edge NEXAFS spectra of the unexposed PTBOCST polymer and of its UV exposed and 

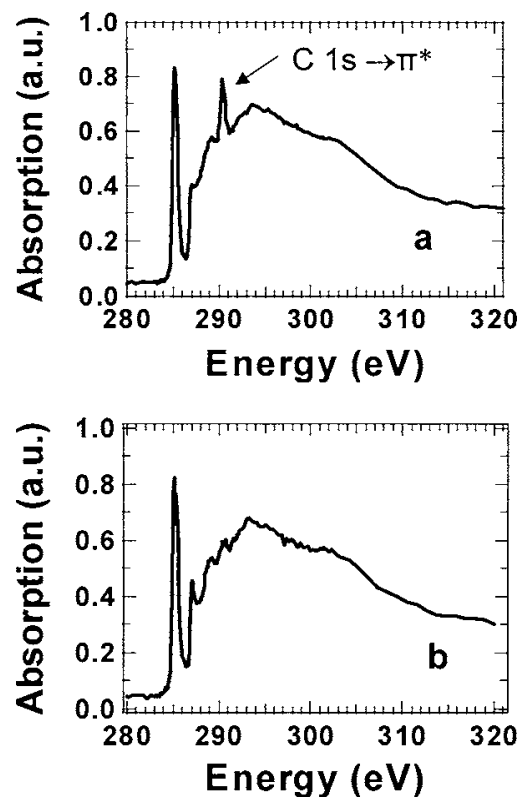

FIG. 3. (a) Carbon $K$ shell NEXAFS spectrum of PTBOCST. The feature at $290.5 \mathrm{eV}$ is attributed to the $\mathrm{C} 1 s \rightarrow \pi^{*}$ transition in the carbonyl group. (b) Carbon $K$ shell NEXAFS spectrum of the deprotected polymer PHOST. Note the almost complete disappearance of the 290.5 peak as the polymer is depleted of the carbonyl group following the deprotection reaction.

deprotected form. Both spectra display a prominent isolated sharp peak at $285.3 \mathrm{eV}$, arising from the carbon $1 s \rightarrow \pi^{*}$ transition in the $\mathrm{C}=\mathrm{C}$ bond. The shoulders between 287 and 290 $\mathrm{eV}$ are due to $1 s \rightarrow \sigma^{*}$ transitions from carbon in $\mathrm{CH}, \mathrm{CH}_{2}$, and $\mathrm{CH}_{3}$ bonds. The prominent peak at $290.5 \mathrm{eV}$ in the unexposed PTBOCST that disappears after the deprotection reaction is attributed to the $\mathrm{C} 1 s \rightarrow \pi^{*}$ transition of the $\mathrm{C}=\mathrm{O}$ molecular orbital in the tert-butoxylcarbonyl group. This particular spectral feature enables chemical contrast in the PTBOCST photoresist to be imaged and quantified. The peak at $287.1 \mathrm{eV}$, which becomes more prominent in the spectrum of the deprotected polymer, is presumably due to the formation of the phenolic $\mathrm{OH}$ group. ${ }^{25}$ Figure 4 depicts images taken at three different energies of a PTBOCST polymer film patterned with the nominal line/space pattern described above. At each photon energy the sample is scanned laterally through the $\mathrm{x}$-ray focus yielding a transverse $x y$ image of the polymer film. The dwell time in Fig. 4 is $1.6 \mathrm{~ms}$ per $50 \mathrm{~nm}$ pixel. The acquisition time for one $10 \mu \mathrm{m} \times 10 \mu \mathrm{m}$ image (not displayed fully in Fig. 4), at this dwell time, is $8 \mathrm{~min}$. The $50 \mathrm{~nm}$ distance per pixel was chosen to reduce radiation exposure while maximizing the spatial resolution. The spatial resolution of the microscope has been tested ${ }^{24}$ on samples with patterns of lines/spaces fabricated in gold with electron beam lithography techniques and supported on $\mathrm{Si}_{3} \mathrm{~N}_{4}$ membranes. Lines (width $35 \mathrm{~nm}$ ) are easily resolved using a zone plate with a $40 \mathrm{~nm}$ outermost zone width. ${ }^{24}$

Figure 4(a) shows an image obtained at $290.5 \mathrm{eV}$. The dark region (zero absorbance) in the lower right-hand corner is due to a hole in the sample. Such holes are used to measure the incident photon flux intensity, $I_{0}$ which is necessary to convert the intensity measured through the polymer

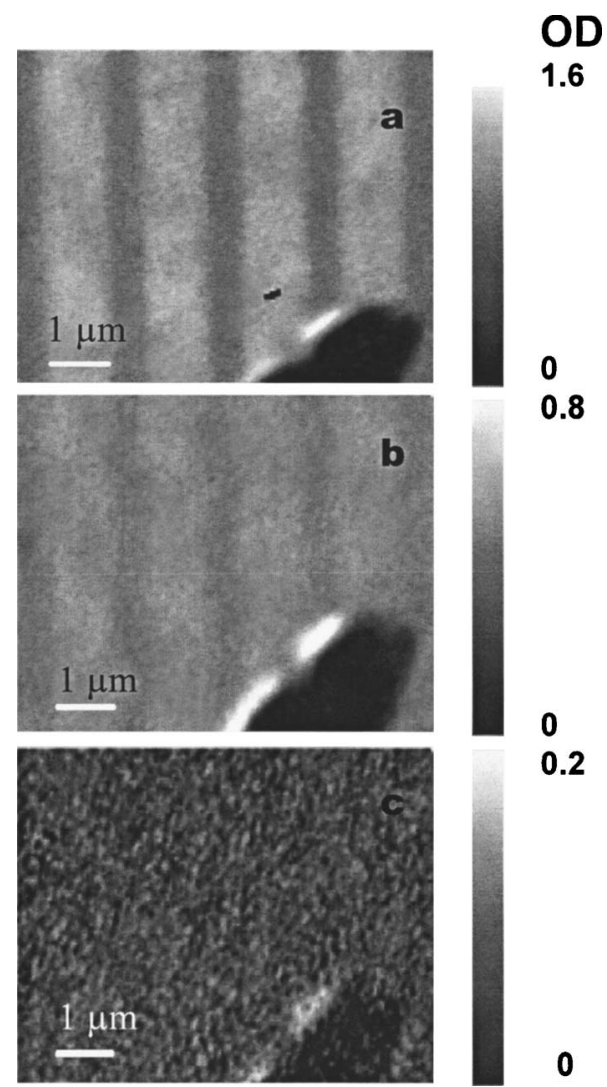

FIG. 4. Transverse $x y$ images (absorption mode) of the patterned PTBOCST sample at (a) $290.5 \mathrm{eV}$, (b) $314.0 \mathrm{eV}$, and (c) $280.0 \mathrm{eV}$. Acquisition time of a $10 \mu \mathrm{m} \times 10 \mu \mathrm{m}$ image (not shown fully) was $8 \mathrm{~min}$, with a dwell time of $1.6 \mathrm{~ms}$ per $50 \mathrm{~nm}$ scan distance. The dark spot in the lower right-hand corner is a hole in the sample where the incident photon flux $I_{0}$ is measured.

sample into optical density. The additional bright features next to the dark spot indicate high absorption at that position. A possible explanation might be increased thickening of the polymer film possibly arising by folding of the film during the formation of the nearby hole during sample mounting onto the TEM grid. Topographical features contribute to the total contrast in STXM images due to the direct proportionality between the x-ray absorption and the number of absorptive centers. In these experiments the number of absorptive centers decreases following the deprotection reaction in the exposed regions due to the release of volatile carboncontaining byproducts. Figure 4(b) shows an image at 314.5 $\mathrm{eV}$ where the main contribution to the contrast originates from this topographical (thickness) difference of our samples. However the image at $280.0 \mathrm{eV}$ (on the same intensity scale), which is below the onset of the $\mathrm{C} K$ edge, displays no such contrast [Fig. 4(c)].

A one-dimensional profile obtained by averaging along the longitudinal direction of the line pattern, obtained by summing the lines in the image at $290.5 \mathrm{eV}$ and plotting along the transverse direction of the pattern, is presented in Fig. 5. The estimated contrast from the line profile, using the formula $\left(I_{\max }-I_{\min }\right) /\left[\left(I_{\max }+I_{\min }\right) / 2\right]$, is $(32.6 \pm 1.1) \%$. $I_{\max }\left(I_{\min }\right)$ is the maximum (minimum) optical density measured in the one-dimensional profile. The image contrast at 


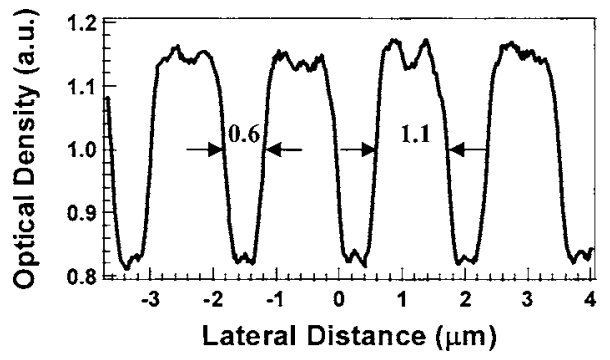

FIG. 5. Line profile of the patterned PTBOCST sample obtained by averaging in the longitudinal direction of the pattern in the image shown in Fig. 3(a).

$310 \mathrm{eV}$, which originates primarily from the topographical contribution is $\approx(14.1 \pm 1.5) \%$. The difference of about $(18.5 \pm 2.6) \%$ represents the chemical contrast contribution to the total contrast of the image recorded at $290.5 \mathrm{eV}$. The uncertainties were estimated as 0.06 for $I_{\max }$ and 0.02 for $I_{\min }$. Part of this uncertainty arises from small variations in the initial sample thickness. The chemical map at $290.5 \mathrm{eV}$ is important in this study since it exhibits the advancement of the direct chemical changes produced by the deprotection reaction. Furthermore, a quantitative spatial distribution of the deprotected polymer after post-exposure bake determines the quality of the pattern transfer during the dissolution step, since dissolution is directly related to the percentage of carbonyl bonds that have been transformed by the deprotection. $^{26}$

The quantitative mapping of the carbonyl group can be obtained with the least-squares method by determining the coefficients $a_{x, y, n}^{\mathrm{PTBOCST}}$ and $a_{x, y, n}^{\mathrm{PHOST}}$ in the following equation that minimizes the quantity $\left[\mathrm{OD}_{x, y, n}^{\text {measured }}-\mathrm{OD}_{x, y, n}^{\text {calculated }}\right]^{2}$ :

$$
\begin{aligned}
\mathrm{OD}_{x, y, n}^{\text {calculated }}= & \text { const }_{n}+a_{x, y, n}^{\mathrm{PTBOCST}} \mathrm{OD}_{\mathrm{PTBOCST}, n} \\
& +a_{x, y, n}^{\mathrm{PHOST}_{\mathrm{OD}}} \mathrm{OD}_{\mathrm{PHOST}, n},
\end{aligned}
$$

where $\mathrm{OD}_{x, y, n}^{\text {measured }}$ is the optical density at each pixel $(x, y)$ of the recorded images at different energies $(1, \ldots, n)$ in the measured stack, OD PTBOCST, $n_{\text {and } \mathrm{OD}_{\mathrm{PHOST}, n} \text { are reference }}$ spectra of the pure polymers [PTBOCST (unexposed) and (PHOST) deprotected]. The measured spectrum at each pixel is thus a linear combination of reference spectra of pure polymers and the linear coefficients are quantitative measures of each pure component at each position. If the reference spectra are in OD from polymer films having the same thickness as the corresponding regions of the patterned sample, then the coefficient $a_{x, y, n}^{\text {PTBOCST }} \times 100$ represents the fraction of unexposed polymer at each position measured as a percentage. Similarly, $a_{x, y, n}^{\mathrm{PHOST}} \times 100$ gives the fraction of the exposed and deprotected polymer at each position. Normally, $a_{x, y, n}^{\mathrm{PTBOCST}}$ and $a_{x, y, n}^{\mathrm{PHOST}}$ have values between 0 and 1 and at each pixel $(x, y)$ and their sum equals 1 . Due to measurement statistics, the coefficients can be less then zero or greater then 1. For example, if the OD of the reference is much weaker than that in an image of a sample of unknown composition, then the coefficients can be higher than 1 . The

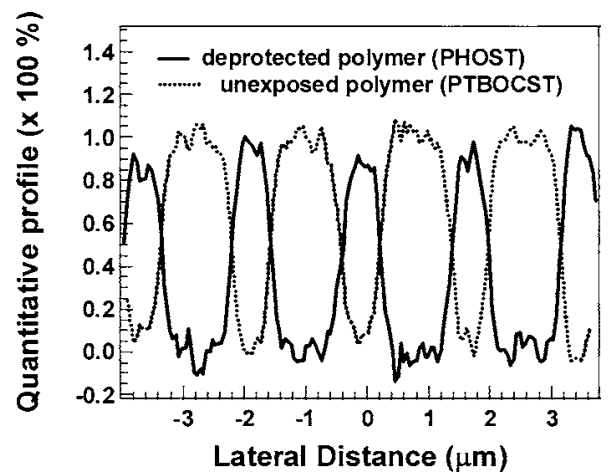

FIG. 6. Spatial distribution of the fraction of carbonyl group (unexposed polymer): dotted line, and of the deprotected polymer: solid line, present after the post-exposure bake stage.

constant in Eq. (2) accounts for a difference in energyindependent scattering of the $\mathrm{x}$ rays.

At $290.5 \mathrm{eV}, a_{x, y, n}^{\mathrm{PTOCST}}$ represents the spatial distribution of the PTBOCST carbonyl group, which we normally identify with the unexposed polymer. If the reference spectra are scaled per unit thickness (representing OD per one nm thickness), using the atomic scattering factors, ${ }^{27}$ then the linear coefficients are obtained in units of nanometers and their maximum values (occurring in the corresponding centers of each polymer region) represent the thickness of the unexposed and post-exposure deprotected areas at the centers of each polymer. By using this procedure we estimated the thickness of the unexposed and deprotected regions to be approximately 130 and $100 \mathrm{~nm}$, respectively. These values are in agreement with the thickness values estimated during the polymer film fabrication. Figure 6 presents the quantitative map profile of the unexposed and post-exposure deprotected polymers and shows the percentage of the initial PTBOCST polymer that is converted into the deprotected form PHOST at each position in the imaged pattern, thus providing a quantitative spatial extent of deprotection. From the quantitative map profile (Fig. 6) it can be seen that most exposed regions contain about $10 \% \pm 5 \%$ of the initial carbonyl groups present even in the center region of the line, while fewer exposed regions show complete deprotection. The presence of carbonyl groups in the exposed area suggests that the deprotection reaction is not complete throughout the exposed regions. Furthermore, the small peak remaining at $290.5 \mathrm{eV}$, which is attributed to the PTBOCST carbonyl group and was confirmed in the measured oxygen spectra (data not shown), does not completely disappear in the spectrum of the fully exposed region of the photoresist [Fig. 3(b)]. Either the deprotection reaction is not complete or residual carbonyl species are not released and remain trapped in the polymer matrix. In addition, the quantitative map profile in Fig. 6 yields information about the sharpness of the imprinted lines after the post exposure bake. It appears that there is a constant finite spatial gradient of the degree of deprotection, since the edges of the imprinted lines are not 
sharply defined at this stage, but rather there is a region of about $400 \pm 50 \mathrm{~nm}$ over which the degree of deprotection steadily varies from zero to the maximum.

Figure 5 shows that the phototreatment and the chemical changes during the high temperature bake have imprinted lines of nominal width of $\sim 0.6 \mu \mathrm{m}$ corresponding to the valleys, whereas the unexposed regions corresponding to the peaks are wider $(\sim 1.1 \mu \mathrm{m})$. The same dimensions of the line pattern are displayed by the quantitative map of Fig. 6 . The exposed regions of the polymer appear to be slightly narrower than the open areas measured on the mask, while the dimension of the unexposed areas is consistent with the measured dimension of the opaque regions of the mask. A possible explanation for this difference in the exposed line dimensions can be the physical volume shrinkage of the film upon deprotection, which could manifest itself not only in thickness, but also in lateral dimension, particularly since the polymer is floated off the substrate. This might be suggested by the fact that approximately $40 \%$ volume shrinkage is expected to occur during the deprotection reaction, whereas only about $23 \%$ thickness shrinkage results from a quantitative analysis. Similar effects were also observed in chemical imaging of similar photoresists by optical techniques such as CARS microscopy ${ }^{15}$ and IR NSOM..$^{10,11}$ In imaging by IR NSOM, the narrowing of the valleys (the exposed regions of the polymer) was in part attributed to the limited reach of the probing IR light at the edges of the deep regions due to the conical shape of the tip. ${ }^{10,11}$ However, this is not the case in the present experiment which does not employ a tip.

A weak periodic pattern superimposed on the profile of the unexposed regions is observed in Figs. 5 and 6. This has also been observed before in samples patterned by exposure through a mask and has been attributed to interference of light leaking under the mask into the unexposed regions. ${ }^{10}$

Chemical changes between the exposed regions of the patterned photoresist and the nonexposed regions include loss of a carbonyl group and the formation of a phenolic $\mathrm{OH}$ group. In addition, the fluorine containing photoacid generator may decompose or diffuse, creating a spatial distribution of the photoacid generator. Both of these regions offer additional opportunities to probe the polymer chemistry by STXM. Experiments to measure pure chemical contrasts at the oxygen $K$ edge $(525-550 \mathrm{eV})$ and the fluorine $K$ edge (680-710 eV) would in principle provide important complementary information to the carbon edge region. Contrast images (due to differences in sample thickness between the exposed and unexposed regions) were observed at both the fluorine and oxygen edges and displayed line/space dimensions consistent with those observed at the carbon edges. The signal-to-noise observed in the oxygen spectra was weaker than that observed at the $\mathrm{C} K$ edge. Therefore we did not attempt chemical quantification at the oxygen $K$ edge. Absorption $(\sim 532.7 \mathrm{eV})$ corresponding to the $\mathrm{O} 1 s \rightarrow \pi^{*}$ transition in the carbonyl group ${ }^{25}$ was observed in both the spectra of the exposed and unexposed regions of the patterned photoresist. The appearance of a small shoulder at $\sim 535.7 \mathrm{eV}$ in the exposed sample, is probably due to the

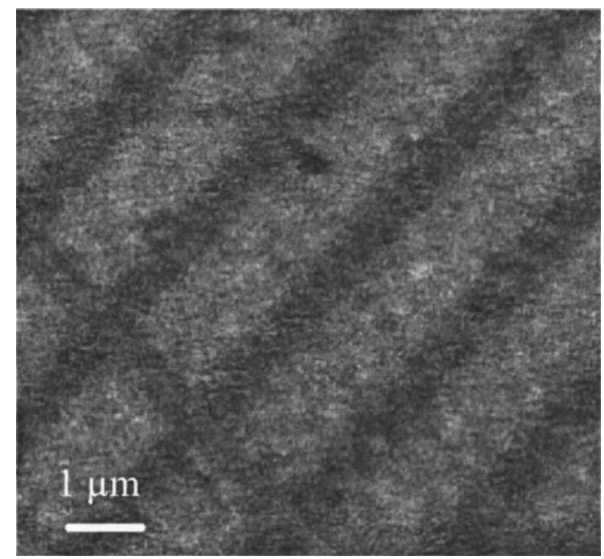

FIG. 7. Transverse image (absorption mode) of a patterned PTBOCST sample damaged by repeatedly scanning a line perpendicular to the patterned lines.

$1 s \rightarrow \sigma^{*}$ transition of the $\mathrm{C}-\mathrm{O}$ in the alcohol group formed upon deprotection. We were unable to identify a fluorine spectrum from these samples. This could be due to the low acid concentration combined with a homogeneous distribution of the acid.

Due to the high energy deposition into small volume, the achievement of high spatial resolution, by focusing the x-ray beam to a spot $(\sim 35 \mathrm{~nm})$, is limited by radiation damage. Radiation damage is primarily manifested by breaking of chemical bonds and loss of mass. ${ }^{28}$ At the same time, in acid catalyzed photoresist chemistry, the x-ray radiation can initiate the acid catalysis reactions, which in the case of PTBOCST photoresist depletes the polymer of the carbonyl group. The decrease in bond density will be manifested as a decrease in the corresponding peak heights in the NEXAFS spectra. ${ }^{29}$ Basic considerations of image contrast, spatial resolution, and radiation damage have to be correlated in choosing the exposure parameters. Acquisitions of images at a large number of energies are particularly subject to radiation damage compared with the acquisition of a single image. Figure 7 shows an example of radiation damage produced by making an $\mathrm{x}$-ray line scan perpendicular to the original line pattern of the sample. The line scan is recorded in the energy range from 275 to $320 \mathrm{eV}$ in steps of $0.4 \mathrm{eV}$ with a dwell time of $3 \mathrm{~ms}$ per $10 \mathrm{~nm}$ scan distance. The repeatedly irradiated line (scanned about 110 times) has the same appearance and has the same spectrum (data not shown) as the deprotected regions of the photoresist due to the depletion of $\mathrm{C}=\mathrm{O}$ bonds. Considering an average photon flux of about $10^{6}$ photons/s at an average photon energy of $300 \mathrm{eV}$, the estimated integrated exposure dose is about $20 \mathrm{~J} / \mathrm{cm}^{2}$. The actual irradiation dose applied in this scan is higher by a factor of $\sim 3$ due to timing overhead in scanning a diagonal rather than a horizontal line. The resulting exposure dose is about 180 times higher than a typical exposure dose used for UV patterning of photoresists. Further experiments, where a reduced number of images in a stack were taken, and with typical dwell times of about $1 \mathrm{~ms}$ per $50 \mathrm{~nm}$ pixel size, showed no observable radiation damage in 
PTBOCST samples. For a dwell time of $1 \mathrm{~ms}$ per $50 \mathrm{~nm}$ scan distance, the radiation exposure at an average energy of 300 $\mathrm{eV}$, with an average photon flux of $10^{6}$ photons/s, in recording a stack at five energies is $10 \mathrm{~mJ} / \mathrm{cm}^{2}$. This is ten times smaller then a typical irradiation exposure dose used in UV patterning of photoresists. However, due to the different interactions of the UV and $\mathrm{x}$-ray radiation with the samples, a direct comparison between $\mathrm{X}$-ray and UV radiation may not be relevant.

\section{SUMMARY}

In this study, we have used STXM as a potential technique for investigating chemically amplified photoresists. Chemical imaging of polymer photoresists using STXM offers several advantages over existing optical techniques. Similar to optical techniques such as IR NSOM, spontaneous Raman microscopy, and CARS, STXM achieves chemical selectivity without the use of fluorescent contrast agents. As shown in this preliminary study, the potential strength of imaging using STXM is based both on chemical contrast and the spatial resolution, which exceeds that of the other spectroscopic techniques, in addition to quantitative measurements of the chemical components at each volume position in the sample.

We measured the spatial distribution of the carbonyl fraction remaining after the post-exposure bake stage with a spatial resolution of $35 \mathrm{~nm}$. This information reveals the effectiveness of the deprotection reaction at different positions in the patterned sample and can be used to theoretically predict the pattern after the dissolution step. ${ }^{25}$ Furthermore, the quantitative analysis independently estimates the thickness of the two regions of the patterned photoresist providing information on the extent of deprotection.

STXM offers the possibility of obtaining chemical images $\left(10 \times 10\right.$ microns $\left.^{2}\right)$ at very high spatial resolution $(50 \mathrm{~nm})$ in just a few minutes and can be adapted to a wide range of sample environments, such as magnetic fields, ${ }^{30}$ variable temperature, ${ }^{31}$ and aqueous media for polymeric and environmental samples. ${ }^{32}$ Furthermore, the near edge $\mathrm{x}$-ray absorption cross section is polarization dependent. ${ }^{21,33}$ The resulting x-ray linear dichroism can be used to image the orientation of specific bonds in (partially) ordered samples. ${ }^{34}$

In conclusion, the chemical sensitivity of STXM allows imaging of polymeric photoresists with chemical contrast. STXM exhibits high spatial resolution and enables fast image acquisition rates. All of these aspects are key in investigating chemically amplified polymer photoresists, promoting STXM as a potentially powerful technique for studying these systems.

\section{ACKNOWLEDGMENTS}

These experiments would not have been possible without the assistance and support of T. Tyliszczak, T. Araki, and D. K. Shuh. M.K.G. acknowledges a Laboratory Directed Research Development grant. L.M., R.P., and S.R.L. acknowledge support by National Science Foundation Grant Nos.
DMR-0302446 and EEC-0310717. R.P. was provided support from Ecole Normale Supérieure de Paris. This work was also supported by the Director, Office of Science, Office of Basic Energy Sciences, Division of Chemical Sciences, Geosciences, and Biosciences and the Division of Materials Sciences of the U. S. Department of Energy at the ALS and LBNL under Contract No. DE-AC03-76S00098.

${ }^{1}$ J. Canning, J. Vac. Sci. Technol. B 15, 2109 (1997).

${ }^{2}$ J. Silverman, J. Vac. Sci. Technol. B 15, 2117 (1997).

${ }^{3}$ M. McCord, J. Vac. Sci. Technol. B 15, 2125 (1997).

${ }^{4}$ L. Harriott, J. Vac. Sci. Technol. B 15, 2130 (1997).

${ }^{5}$ G. Gross, J. Vac. Sci. Technol. B 15, 2136 (1997).

${ }^{6}$ W. Hinsberg, F. A. Houle, J. Hoffnagle, M. Sanchez, G. Walraff, M. Morrison, and S. Frank, J. Vac. Sci. Technol. B 16, 3689 (1998).

${ }^{7}$ P. M. Dentiger, B. Lu, J. W. Taylor, S. J. Bukofsky, G. D. Feke, D. Hessman, and R. D. Grober, J. Vac. Sci. Technol. B 14, 4239 (1996).

${ }^{8}$ B. Lu, J. W. Taylor, F. Cerrina, C. P. Soo, and A. J. Bourdillon, J. Vac. Sci. Technol. B 17, 3345 (1999).

${ }^{9}$ B. Dragnea and S. R. Leone, J. Appl. Phys. 86, 2795 (1999).

${ }^{10}$ B. Dragnea, J. Preusser, J. M. Szarko, L. A. McDonough, and S. R. Leone, Appl. Surf. Sci. 19, 783 (2001).

${ }^{11}$ B. Dragnea, J. Preusser, J. M. Szarko, S. R. Leone, and W. D. Hinsberg, J. Vac. Sci. Technol. B 19, 142 (2001).

${ }^{12}$ B. Dragnea and S. R. Leone, Int. Rev. Phys. Chem. 20, 59 (2001).

${ }^{13}$ F. Zenhausern, M. P. O'Boyle, and H. K. Wickramasinghe, Appl. Phys. Lett. 65, 1623 (1994).

${ }^{14}$ B. Knoll and F. Keilmann, Nature (London) 399, 134 (1999).

${ }^{15}$ E. O. Potma, X. S. Xie, L. Muntean, J. Preusser, D. Jones, Y. Jun, S. R. Leone, W. D. Hinsberg, and S. Wolfgang, J. Phys. Chem. B 108, 1296 (2004).

${ }^{16}$ E. K. Lin, C. L. Soles, D. L. Goldfarb, B. C. Trinque, S. D. Burns, R. L. Jones, J. L. Lenhart, M. Angelopoulos, C. G. Willson, S. K. Satija, and W. L. Wu, Science 297, 372 (2002).

${ }^{17}$ H. Ade, X. Zhang, S. Cameron, C. Costello, J. Kirz, and S. Williams, Science 258, 972 (1992).

${ }^{18}$ H. Ade and B. Hsiao, Science 262, 1427 (1993).

${ }^{19}$ H. Ade, A. P. Smith, H. Zhang, B. Winn, J. Kirz, E. Rightor, and A. P. Hitchcock, J. Electron Spectrosc. Relat. Phenom. 84, 53 (1997).

${ }^{20}$ T. Warwick, H. Padmore, H. Ade, A. P. Hitchcock, E. G. Rightor, and B. Tonner. J. Electron Spectrosc. Relat. Phenom. 84, 85 (1997).

${ }^{21}$ J. Stöhr, NEXAFS Spectroscopy (Springer, Berlin, 1992).

${ }^{22}$ H. Ade and S. G. Urquhart, in Chemical Applications of Synchrotron Radiation, edited by T. K. Sham (World Scientific, Singapore, 2001).

${ }^{23}$ T. Warwick, H. Ade, A. L. D. Kilcoyne, M. Kritscher, T. Tylisczcak, S. Fakra, A. P. Hitchcock, P. Hitchcock, and H. A. Padmore, J. Synchrotron Radiat. 9, 254 (2002).

${ }^{24}$ A. L. Kilcoyne, T. Tyliszczak, W. F. Steele, S. Fakra, P. Hitchcock, K. Franck, E. Anderson, B. Harteneck, E. G. Rightor, G. E. Mitchell, A. P. Hitchcock, L. Yang, T. Warwick, and H. Ade, J. Synchrotron Radiat. 10, 125 (2002).

${ }^{25}$ S. C. B. Myneni, Rev. Mineralogy Geochem. 49, 485 (2002).

${ }^{26}$ F. A. Houle, W. D. Hinsberg, and M. I. Sanchez, Macromolecules 35, 8591 (2002).

${ }^{27}$ B. L. Henke, P. Lee, T. J. Tanaka, R. L. Shimabukuro, and B. K. Fujikawa, At. Data Nucl. Data Tables 27, 1 (1982).

${ }^{28}$ T. Coffey, S. G. Urquhart, and H. Ade, J. Electron Spectrosc. Relat. Phenom. 122, 65 (2002).

${ }^{29}$ T. Beetz and C. Jacobsen, J. Synchrotron Radiat. 10, 280 (2002).

${ }^{30}$ S. K. Kim, J. B. Kortright, and S. C. Shin, Appl. Phys. Lett. 78, 2742 (2001).

${ }^{31}$ Y. Wang, C. Jacobsen, J. Maser, and A. Osanna, J. Microsc. 197, 80 (2000).

${ }^{32}$ U. Neuhausler, S. Abend, C. Jacobsen, and G. Lagaly, Colloid Polym. Sci. 277, 719 (1999).

${ }^{33}$ F. Sette, J. Stöhr, and A. P. Hitchcock, J. Chem. Phys. 81, 4906 (1984).

${ }^{34}$ H. Ade, A. P. Smith, S. Cameron, R. Cieslinski, G. Mitchell, B. Hsiao, and E. Rightor, Polymer 36, 1843 (1995). 